\title{
Reversing entanglement change by a weak measurement
}

\author{
Qingqing Sun, ${ }^{1, *}$ M. Al-Amri, ${ }^{2}$ Luiz Davidovich, ${ }^{3}$ and M. Suhail Zubairy ${ }^{1}$ \\ ${ }^{1}$ Department of Physics and Institute for Quantum Science and Engineering, Texas A\&M University, College Station, Texas 77843, USA \\ ${ }^{2}$ National Centre for Mathematics and Physics, KACST, P.O. Box 6086, Riyadh 11442, Saudi Arabia \\ ${ }^{3}$ Instituto de Física, Universidade Federal do Rio de Janeiro, 21941-972 Rio de Janeiro, Brazil
}

(Received 17 August 2010; published 19 November 2010)

\begin{abstract}
Entanglement of a system changes due to interactions with the environment. A typical type of interaction is amplitude damping. If we add a detector to monitor the environment and only select the no-damping outcome, this amplitude damping is modified into a weak measurement. Here we show that the entanglement change of a twoqubit state due to amplitude damping or weak measurement can be probabilistically reversed. For the amplitudedamping case, the entanglement partially recovers under most conditions. For the weak-measurement case, the recovery of the initial entangled state is exact. The reversal procedure involves another weak measurement, preceded and followed by bit flips applied to both qubits. We propose a linear optics scheme for the experimental demonstration of these procedures.
\end{abstract}

DOI: 10.1103/PhysRevA.82.052323

PACS number(s): 03.67.Bg, 03.65.Yz, 42.50.Ex, 03.65.Ta

\section{INTRODUCTION}

Quantum entanglement is the key resource for quantum information processing. However, entanglement is usually affected by the interaction with the environment, so it is very important to preserve the entanglement against environmental noise. Several methods have been suggested for quantum state protection. For example, quantum error correcting codes use auxiliary qubits to store information, assuming each qubit interacts with its environment independently [1]. After interaction a multiqubit measurement shows which error occurred and a reversal procedure can be applied correspondingly. Another strategy is to have qubits symmetrically coupled with the same environment [2]. There is then a linear subspace decoupled from the environment and any state in that subspace is decoherence free. The third method is to use a controlled series of pulses with a repetition interval comparable to the environment memory time, which washes out the decoherence effect [3].

Recently, it was pointed out that a weak measurement, together with bit flips, protects quantum states of a singlepartite system [4]. For weak measurements [5], the outcome cannot determine the state of the measured system precisely and therefore does not totally collapse the state of the system. If the full information of the initial state has been passed over to the final state, then it should be possible in principle to recover the initial state by implementing a sequence of operations. For deterministic reversible measurements, the initial state lies in a certain subspace and the measurement provides no information about it $[6,7]$. In this case, it is always possible to reverse the dynamics and recover the initial state. On the other hand, for probabilistically reversible measurements [8-15], only certain outcomes of the measurement keep the full information of the initial state and are possible to reverse. Probabilistic reversal with a weak measurement has already been demonstrated on a superconducting phase qubit [14], as well as on a photonic qubit [16].

In this paper, we propose using a weak measurement to reverse the entanglement change of two qubits. The initially

*qsun@physics.tamu.edu entangled qubits go through separate paths and interact with their own individual and independent environments, gradually changing their entanglement. When we decide to recover the entanglement, we apply first a bit-flipping operation to both qubits, then perform a null-result weak measurement, and finally flip the qubits back. We specifically consider the entanglement change due to two types of system-environment interactions. One is amplitude damping, which always lowers the entanglement. In this case the reversal procedure partially recovers the entanglement under most conditions. The other is amplitude damping with monitoring of the environment, which is essentially a weak measurement. The entanglement can be either increased or decreased, but the reversal procedure restores the initial entangled state and the entanglement exactly. This is allowed, even though all these operations are local operations, due to the probabilistic nature of the method.

This paper is organized as follows: First we show the state maps of a single qubit for the two interaction types. Then in each case we discuss the entanglement dynamics of two qubits, followed by the reversal analysis and the conditions for successful recovery. Finally, we propose a linear optical scheme for the implementation of our procedure.

\section{THEORY}

\section{A. State map for a single qubit}

The two main operations in our scheme are amplitude damping and weak measurement. Here we discuss their state maps in the single-qubit case for better understanding. Taking a two-level atom as an example, if the environment is a vacuum with zero temperature, amplitude damping corresponds to the following map [17]:

$$
\begin{gathered}
|0\rangle_{S}|0\rangle_{E} \rightarrow|0\rangle_{S}|0\rangle_{E}, \\
|1\rangle_{S}|0\rangle_{E} \rightarrow \sqrt{1-p}|1\rangle_{S}|0\rangle_{E}+\sqrt{p}|0\rangle_{S}|1\rangle_{E},
\end{gathered}
$$

where $p \in[0,1]$ is the probability of losing the system excitation into the environment. Within the Weisskopf-Wigner approximation, the probability $1-p$ of finding the atom in the excited state decreases exponentially with time. We do not 
display the time dependence explicitly both for simplicity and generality. Of course, if there is no initial excitation in both the system and the environment, the combined state of the system will not evolve.

A null-result weak measurement is different from amplitude damping, in the sense that we add an ideal detector to monitor the environment. Whenever there is an excitation in the environment we discard the result. This postselection removes the $\sqrt{p}|0\rangle_{S}|1\rangle_{E}$ term from the state map. The resulting map is

$$
\begin{gathered}
|0\rangle_{S}|0\rangle_{E} \rightarrow|0\rangle_{S}|0\rangle_{E}, \\
|1\rangle_{S}|0\rangle_{E} \rightarrow \sqrt{1-p}|1\rangle_{S}|0\rangle_{E},
\end{gathered}
$$

where in the second line the state after mapping has a norm less than 1, corresponding to the finite success probability for the weak measurement. When we trace out the environment, the system maps as

$$
|i\rangle_{S} \rightarrow(1-p)^{i / 2}|i\rangle_{S}, \quad(i=0,1)
$$

Using these relations we now consider two-qubit states.

\section{B. Weak measurement and its reversal}

In this subsection we analyze the entanglement change due to a weak measurement and its reversal. For two entangled qubits having independent weak measurements as in Eq. (3), the density matrix evolves as

$$
\sum_{i j k l} \rho_{i j k l}|i j\rangle_{S}\left\langle\left. k l\right|_{S} \rightarrow \sum_{i j k l}(1-p)^{(i+j+k+l) / 2} \rho_{i j k l} \mid i j\right\rangle_{S}\left\langle\left. k l\right|_{S}\right.
$$

where $i, j, k, l=0,1$. The entanglement can increase or decrease during this process. This is illustrated by Salles et al. [18] with an example of an initial pure state $\alpha|00\rangle+$ $\delta|11\rangle$. After weak measurements, the normalized state is $[\alpha|00\rangle+(1-p) \delta|11\rangle] / \sqrt{|\alpha|^{2}+(1-p)^{2}|\delta|^{2}}$, with a raised $|00\rangle$ weight and a lowered $|11\rangle$ weight. If initially $|\alpha|>|\delta|$, after weak measurements the two weights are more uneven, which decreases the entanglement. But if initially $|\alpha|<|\delta|$, after weak measurements the two weights get closer and the entanglement increases with $p$. At $p=1-|\alpha| /|\delta|$, the two components have equal weights, which leads to a maximally entangled state. For larger $p$, the $|00\rangle$ component weighs more than the $|11\rangle$ component, so the entanglement starts decreasing with $p$.

To recover the entanglement change, we apply the following procedure [4]: for each qubit, first a bit flip to swap the states $|0\rangle_{S} \rightarrow|1\rangle_{S}$, then another null-result weak measurement with the same $p$, and finally another bit flip back. The density matrix evolves as

$$
\begin{aligned}
& \sum_{i j k l}(1-p)^{(i+j+k+l) / 2} \rho_{i j k l}|i j\rangle_{S}\left\langle\left. k l\right|_{S}\right. \\
& \quad \rightarrow \sum_{i j k l}(1-p)^{(i+j+k+l) / 2} \rho_{i j k l}|1-i, 1-j\rangle_{S}\left\langle 1-k, 1-\left.l\right|_{S}\right. \\
& \quad \rightarrow \sum_{i j k l}(1-p)^{2} \rho_{i j k l}|1-i, 1-j\rangle_{S}\left\langle 1-k, 1-\left.l\right|_{S}\right. \\
& \quad \rightarrow \sum_{i j k l}(1-p)^{2} \rho_{i j k l}|i j\rangle_{S}\left\langle\left. k l\right|_{S}\right.
\end{aligned}
$$

where the three arrows correspond to bit flip, weak measurement, and bit flip. Due to the two bit flips, the reversal procedure increases the weight of high-excitation components. After normalization we recover the exact initial state, and the common factor of all elements $(1-p)^{2}$ is the success probability for the whole process. Naturally the entanglement is also recovered to the initial value.

\section{Amplitude damping and its reversal}

Here we analyze the alternative situation. For two entangled qubits having independent amplitude damping, each qubit undergoes the dynamics defined by Eq. (1). If initially the system is in a general pure state $\alpha|00\rangle_{S}+\beta|01\rangle_{S}+\gamma|10\rangle_{S}+$ $\delta|11\rangle_{S}$, and both environments are in the ground state, the combined state evolves as

$$
\begin{aligned}
&\left(\alpha|00\rangle_{S}+\beta|01\rangle_{S}+\gamma|10\rangle_{S}+\delta|11\rangle_{S}\right)|00\rangle_{E} \\
& \rightarrow \alpha|00\rangle_{S}|00\rangle_{E}+\beta \sqrt{1-p}|01\rangle_{S}|00\rangle_{E}+\beta \sqrt{p}|00\rangle_{S}|01\rangle_{E} \\
&+\gamma \sqrt{1-p}|10\rangle_{S}|00\rangle_{E}+\gamma \sqrt{p}|00\rangle_{S}|10\rangle_{E} \\
&+\delta(1-p)|11\rangle_{S}|00\rangle_{E}+\delta \sqrt{(1-p) p}|10\rangle_{S}|01\rangle_{E} \\
&+\delta \sqrt{(1-p) p}|01\rangle_{S}|10\rangle_{E}+\delta p|00\rangle_{S}|11\rangle_{E} .
\end{aligned}
$$

In general, from a system-environment combined state $|\psi\rangle_{S E}$, we can obtain the density matrix of the system by tracing out the environment, $\rho_{S}=\operatorname{Tr}_{E}\left(|\psi\rangle_{S E}\left\langle\left.\psi\right|_{S E}\right)\right.$. Therefore the system after damping is described by

$$
\rho_{d}=\left(\begin{array}{cccc}
|\alpha|^{2}+p|\beta|^{2}+p|\gamma|^{2}+p^{2}|\delta|^{2} & (1-p)^{1 / 2} \alpha \beta^{*}+p(1-p)^{1 / 2} \gamma \delta^{*} & (1-p)^{1 / 2} \alpha \gamma^{*}+p(1-p)^{1 / 2} \beta \delta^{*} & (1-p) \alpha \delta^{*} \\
(1-p)^{1 / 2} \beta \alpha^{*}+p(1-p)^{1 / 2} \delta \gamma^{*} & (1-p)|\beta|^{2}+p(1-p)|\delta|^{2} & (1-p) \beta \gamma^{*} & (1-p)^{3 / 2} \beta \delta^{*} \\
(1-p)^{1 / 2} \gamma \alpha^{*}+p(1-p)^{1 / 2} \delta \beta^{*} & (1-p) \gamma \beta^{*} & (1-p)|\gamma|^{2}+p(1-p)|\delta|^{2} & (1-p)^{3 / 2} \gamma \delta^{*} \\
(1-p) \delta \alpha^{*} & (1-p)^{3 / 2} \delta \beta^{*} & (1-p)^{3 / 2} \delta \gamma^{*} & (1-p)^{2}|\delta|^{2}
\end{array}\right) .
$$

Calculating the eigenvalues of $\rho_{d}\left(\sigma_{y} \otimes \sigma_{y}\right) \rho_{d}^{*}\left(\sigma_{y} \otimes \sigma_{y}\right)$ and putting them in a decreasing order, we obtain the concurrence
$C=\max \{0, \Lambda\}$, where $\Lambda=\sqrt{\lambda_{1}}-\sqrt{\lambda_{2}}-\sqrt{\lambda_{3}}-\sqrt{\lambda_{4}}$ [19]. For the initial state, $C=2|\alpha \delta-\beta \gamma|$. After damping it 
drops to

$$
C_{d}(p)=\max \left[0,2(1-p)\left(|\alpha \delta-\beta \gamma|-p|\delta|^{2}\right)\right],
$$

which is less than the initial value and decreases with $p$, so the system entanglement gets smaller due to the amplitude damping. This result was obtained earlier in [20] by explicitly solving the density matrix equations. For $|\alpha \delta-\beta \gamma|<|\delta|^{2}$, the entanglement vanishes at $p=\left|(\alpha \delta-\beta \gamma) / \delta^{2}\right|$, before the excitation in the qubit system damps out. This is the so-called entanglement sudden death (ESD) [21].

To recover the entanglement, we again apply the reversal procedure as in Eq. (5). The final density matrix reads:

$$
\rho_{r}=\left[1+p\left(|\beta|^{2}+|\gamma|^{2}+2|\delta|^{2}\right)+p^{2}|\delta|^{2}\right]^{-1}\left(\begin{array}{cccc}
|\alpha|^{2}+p|\beta|^{2}+p|\gamma|^{2}+p^{2}|\delta|^{2} & \alpha \beta^{*}+p \gamma \delta^{*} & \alpha \gamma^{*}+p \beta \delta^{*} & \alpha \delta^{*} \\
\beta \alpha^{*}+p \delta \gamma^{*} & |\beta|^{2}+p|\delta|^{2} & \beta \gamma^{*} & \beta \delta^{*} \\
\gamma \alpha^{*}+p \delta \beta^{*} & \gamma \beta^{*} & |\gamma|^{2}+p|\delta|^{2} & \gamma \delta^{*} \\
\delta \alpha^{*} & \delta \beta^{*} & \delta \gamma^{*} & |\delta|^{2}
\end{array}\right) .
$$

The success probability for the reversal is

$$
P_{r}(p)=\left[1+p\left(|\beta|^{2}+|\gamma|^{2}+2|\delta|^{2}\right)+p^{2}|\delta|^{2}\right](1-p)^{2} .
$$

It is clear that $P_{r}(p) \geqslant(1-p)^{2}$ for an arbitrary initial state. Therefore the success probability is always higher than the previous case in Sec. II B, but the reversal is not exact.

The concurrence after reversal is

$$
C_{r}(p)=\max \left[0, \frac{2\left(|\alpha \delta-\beta \gamma|-\mathrm{p}|\delta|^{2}\right)}{1+\mathrm{p}\left(1+|\delta|^{2}-|\alpha|^{2}\right)+\mathrm{p}^{2}|\delta|^{2}}\right] .
$$

A careful comparison with the damped concurrence shows several interesting points:
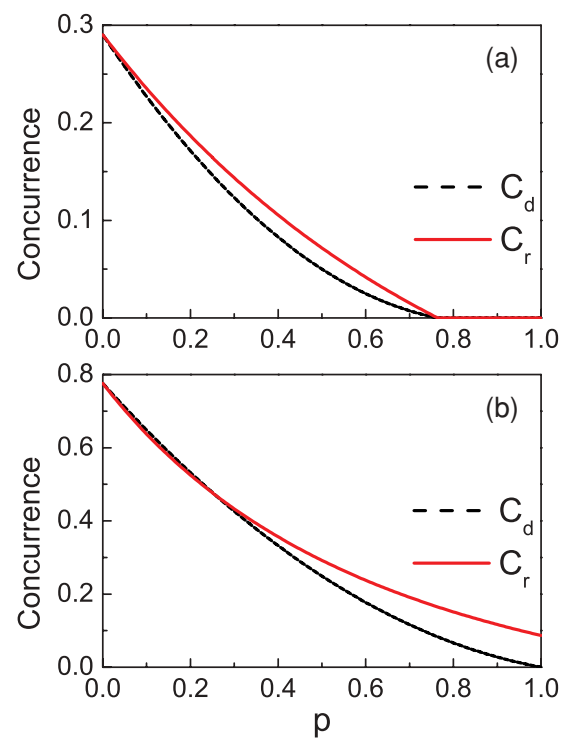

FIG. 1. (Color online) Concurrence as a function of $p$. (a) $\alpha=0.70, \beta=0.40, \gamma=0.40, \delta=0.44$. For $|\alpha| \geqslant|\delta|$, the reversed concurrence $C_{r}$ is always higher than the damped concurrence $C_{d}$. This figure also serves as an example of ESD. Both concurrences go to 0 beyond the ESD point. (b) $\alpha=0.05, \beta=-0.60, \gamma=0.60$, $\delta=0.53$. For $|\alpha|<|\delta|$, the reversed concurrence is higher than the damped concurrence only for large $p$. This figure also serves as an example without ESD. When $p \rightarrow 1, C_{d}$ goes to 0 while $C_{r}$ is finite. (i) $C_{r}$ also bears the same factor $\left(|\alpha \delta-\beta \gamma|-p|\delta|^{2}\right)$, which means that at or beyond the ESD point, the resulting state is also separable and no entanglement is recovered. This is reasonable because all operations are local, and therefore they cannot create entanglement for two distant qubits in a separable state.

(ii) If there is no ESD, the damped concurrence vanishes in the limit $p \rightarrow 1$, while the reversed concurrence approaches a finite value. The price we pay is that the probability for successful recovery is vanishingly small in this limit.

(iii) For an initial state with $|\alpha| \geqslant|\delta|$, the reversed concurrence is always higher than the damped one, as shown in Fig. 1(a). Initially, the lower levels weigh more and damping further increases them; after the reversal the upper levels and the lower levels become more even and the entanglement partially recovers.

(iv) For an initial state with $|\alpha|<|\delta|$, the reversed concurrence is higher than the damped concurrence only for

$$
\begin{aligned}
p> & {\left[\sqrt{\left(|\beta|^{2}+|\gamma|^{2}+3|\delta|^{2}\right)^{2}-4|\delta|^{2}}\right.} \\
& \left.-\left(|\beta|^{2}+|\gamma|^{2}+|\delta|^{2}\right)\right] / 2|\delta|^{2} .
\end{aligned}
$$

An example is shown in Fig. 1(b).

With this general formalism at hand, we discuss two special cases. If the initial state is $\alpha|00\rangle+\delta|11\rangle$, the damped concurrence is $2(1-p)|\delta|(|\alpha|-p|\delta|)$ and the reversed concurrence is $2|\delta|(|\alpha|-p|\delta|) /\left(1+2 p|\delta|^{2}+p^{2}|\delta|^{2}\right)$. For parameters $|\alpha| \geqslant|\delta|$, the entanglement always improves after the previous procedure. If the initial state is $\beta|01\rangle+\gamma|10\rangle$, the damped concurrence is $2(1-p)|\beta \gamma|$ and the reversed concurrence is $2|\beta \gamma| /(1+p)$. Therefore, the entanglement is always partially recovered in this case. In both cases, entanglement recovers as long as the initial state does not allow entanglement sudden death.

If the initial state is a mixed state, all the density matrix elements are variables. There is no explicit expression for the concurrence in this case and we cannot, in general, tell whether the entanglement is recovered.

\section{IMPLEMENTATION WITH LINEAR OPTICS}

Both the amplitude damping and the weak measurement can be implemented in linear optics, using the photon polarization 


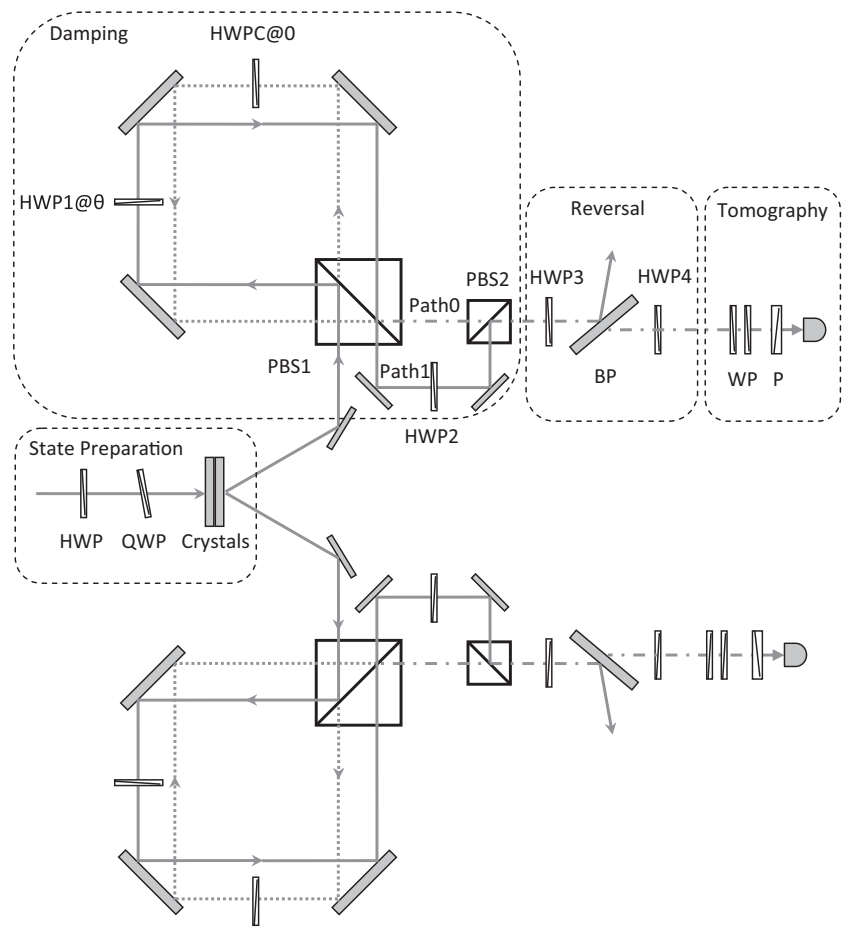

FIG. 2. The linear optics scheme. The entangled photon pair is generated by the crystals. After the interferometer, PBS2 recombines the two outcome paths incoherently to obtain the damped state, or one can use only path 0 to achieve a state having weak measurement. HWP3, BP, and HWP4 compose the reversal process, corresponding to the flip, weak measurement, flip. Finally, the state is measured by quantum tomography.

as a qubit. Here we propose an experimental setup as shown in Fig. 2. The polarization-entangled photon pairs can be produced by two adjacent type-I crystals [22]. The generated state is

$$
|\psi\rangle=|\alpha||H H\rangle+e^{i \delta}|\beta||V V\rangle,
$$

where $H$ means horizontal polarization and $V$ means vertical polarization. Both the coefficients $|\alpha|,|\beta|$ and the relative phase $\delta$ can be controlled by a half-wave plate (HWP) and a tilted quarter-wave plate (QWP) before the crystals. One can also produce photons entangled with orthogonal polarizations:

$$
|\psi\rangle=\left(|H V\rangle+e^{i \delta}|V H\rangle\right) / \sqrt{2}
$$

by rotating the polarization of one of the photons produced by this process, or by type-II phase matching [23], where the down-converted photons are emitted to two cones with different polarizations.

The two photonic qubits are then spatially separated and each goes through a Sagnac-like interferometer that implements the amplitude damping or the weak measurement [24]. We assign the bit value 0 to the $H$ polarization and 1 to the $V$ polarization, and the two outcome paths 0 and 1 represent the number of excitations in the final environment. The incoming photon first encounters a polarization beam splitter (PBS). If the photon has $H$ polarization, it is transmitted through the PBS. After being reflected by the mirrors and going through a 0-deg HWP, HWP0, for optical length matching, this photon is transmitted through the PBS again and leaves the interferometer through the outcome path 0 . The whole process corresponds to the state map $|0\rangle_{S}|0\rangle_{E} \rightarrow|0\rangle_{S}|0\rangle_{E}$. On the other hand, if the photon has $V$ polarization, it is reflected by the PBS and goes along a spatially separated path. On this path HWP1 rotates the polarization to $\cos (2 \theta)|V\rangle+\sin (2 \theta)|H\rangle$. After hitting the PBS again, only the $|V\rangle$ component is reflected to the outcome path 0 , while the $|H\rangle$ component is transmitted along another outcome path 1 . They correspond to the two result terms of the state map $|1\rangle_{S}|0\rangle_{E} \rightarrow$ $\sqrt{1-p}|1\rangle_{S}|0\rangle_{E}+\sqrt{p}|0\rangle_{S}|1\rangle_{E}$, with $p=\sin (2 \theta)^{2}$. We can use a QWP, a HWP, and a PBS to incoherently recombine the two outcome paths and thus obtain the amplitude-damped state. Using the same interferometer setup we can realize weak measurements as well, with the only difference that now we just collect photons from the outcome path 0 . This is equivalent to monitoring the absence of environmental excitation in a weak measurement.

For the reversal procedure we first use a HWP to flip the polarizations [16]. Then a set of Brewster-angle glass plates (BPs) reflects the $V$-polarized photons out with a probability $p$. The $|H\rangle$ state passes the BPs without any loss, while the $|V\rangle$ state becomes $\sqrt{1-p}|V\rangle$ after passing through. This corresponds to the state map of weak measurement described by Eq. (3). Finally, another HWP flips the polarization back. The resulting concurrence is determined by quantum tomography (see [24] for details).

We note that previously a BP filter has also been used for entanglement distillation [25]. Here we are more interested in recovering the entanglement toward the initial value instead of just increasing it. In some cases the reversal procedure decreases the entanglement, as demonstrated by the example in Sec. II B.

\section{CONCLUSION}

In conclusion, we propose a procedure to reverse the entanglement change due to weak measurement or amplitude damping of a two-qubit state and propose a linear optics scheme for implementation. In the weak-measurement case, the initial entangled state and the entanglement recover exactly. In the amplitude-damping case, the concurrence always partially recovers if initially the $|00\rangle$ weight is larger than the $|11\rangle$ weight. Otherwise the damping probability $p$ has to be sufficiently large to achieve the entanglement recovery. If $p$ goes beyond the ESD point, there is no recovery at all because local operations cannot create entanglement. Our procedure works by changing the weight of different terms in the state and filtering the entanglement toward the initial value.

\section{ACKNOWLEDGMENTS}

This work is supported by a grant from the King Abdul Aziz City for Science and Technology (KACST) and an NPRP grant from Qatar National Research Fund (QNRF). M.A. and L.D. gratefully acknowledge the hospitality at Texas A\&M University where this work was done. L.D. acknowledges the support of the Brazilian National Institute of Science and Technology for Quantum Information and of the Brazilian agencies CNPq and FAPERJ. 
[1] P. W. Shor, Phys. Rev. A 52, R2493 (1995); A. M. Steane, Phys. Rev. Lett. 77, 793 (1996); A. R. Calderbank and P. W. Shor, Phys. Rev. A 54, 1098 (1996); A. M. Steane, Proc. R. Soc. London A 452, 2551 (1996).

[2] P. Zanardi and M. Rasetti, Phys. Rev. Lett. 79, 3306 (1997); D. A. Lidar, I. L. Chuang, and K. B. Whaley, ibid. 81, 2594 (1998).

[3] L. Viola and S. Lloyd, Phys. Rev. A 58, 2733 (1998); L. Viola, E. Knill, and S. Lloyd, Phys. Rev. Lett. 82, 2417 (1999); L. Viola, S. Lloyd, and E. Knill, ibid. 83, 4888 (1999).

[4] Q. Sun, M. Al-Amri, and M. S. Zubairy, Phys. Rev. A 80, 033838 (2009).

[5] A. N. Korotkov, Phys. Rev. B 60, 5737 (1999).

[6] H. Mabuchi and P. Zoller, Phys. Rev. Lett. 76, 3108 (1996).

[7] M. A. Nielsen and C. M. Caves, Phys. Rev. A 55, 2547 (1997).

[8] M. Ueda and M. Kitagawa, Phys. Rev. Lett. 68, 3424 (1992).

[9] A. Imamoḡlu, Phys. Rev. A 47, R4577 (1993).

[10] A. Royer, Phys. Rev. Lett. 73, 913 (1994).

[11] M. Ueda, N. Imoto, and H. Nagaoka, Phys. Rev. A 53, 3808 (1996).

[12] M. Ban, J. Phys. A 34, 9669 (2001).
[13] M. Koashi and M. Ueda, Phys. Rev. Lett. 82, 2598 (1999).

[14] N. Katz et al., Phys. Rev. Lett. 101, 200401 (2008).

[15] A. N. Korotkov and A. N. Jordan, Phys. Rev. Lett. 97, 166805 (2006).

[16] Y.-S. Kim, Y.-W. Cho, Y.-S. Ra, and Y.-H. Kim, Opt. Express 17, 11978 (2009).

[17] V. Weisskopf and E. Wigner, Z. Phys. 63, 54 (1930).

[18] A. Salles, F. de Melo, M. P. Almeida, M. Hor-Meyll, S. P. Walborn, P. H. Souto Ribeiro, and L. Davidovich, Phys. Rev. A 78, 022322 (2008).

[19] W. K. Wootters, Phys. Rev. Lett. 80, 2245 (1998).

[20] R. Tahira, M. Ikram, T. Azim, and M. S. Zubairy, J. Phys. B 41, 205501 (2008)

[21] T. Yu and J. H. Eberly, Phys. Rev. Lett. 97, 140403 (2006).

[22] P. G. Kwiat, E. Waks, A. G. White, I. Appelbaum, and P. H. Eberhard, Phys. Rev. A 60, R773 (1999).

[23] P. G. Kwiat, K. Mattle, H. Weinfurter, A. Zeilinger, A. V. Sergienko, and Y. Shih, Phys. Rev. Lett. 75, 4337 (1995).

[24] M. P. Almeida, F. de Melo, M. Hor-Meyll, A. Salles, S. P. Walborn, P. H. S. Ribeiro, and L. Davidovich, Science 316, 579 (2007).

[25] P. G. Kwiat, S. Barraza-Lopes, A. Stefanov, and N. Gisin, Nature (London) 409, 1014 (2001). 